\title{
On-orbit Construction Experiment by Tele-operated Robot Arm
}

\author{
Hiroshi Ueno *, Hitoshi Satoh *, Shigeru Aoki *, Tetsuji Yoshida *, \\ Kohtaro Matsumoto ** and Sachiko Wakabayashi **
}

\author{
*Space Systems Division, Shimizu Corporation \\ Tokyo, Japan \\ Tel:81-3-5441-8951, Fax:81-3-5441-8955, \\ Email: hueno@spo.shimz.co.jp
}

\author{
**National Aerospace Laboratory \\ Tokyo, Japan
}

Tel: 81-422-47-5911, Fax: 81-422-47-8813, Email:matumoto@nal.go.jp

\begin{abstract}
On-orbit assembly technology is a key for future large-scale structure construction. For several decades, many concepts of space structures have been proposed and some of them have the idea of robotic construction systems. Authors have developed a concept of a truss joint system for largescale orbital structures and worked for space robotics research. Participating aJapanese space robotics experiment project on Engineering Test Satellite No. 7 scheduled to be launched by NASDA in 1997, the authors will conduct space truss construction experiments. The experiments have objectives to establish basic technologies of on-orbit assembly work and robotic construction by using NASDA's manipulator on the satellite. Our experiment system consists of the experiment unit including a truss joint and $a$ deployable truss on the satellite, and our teleoperation facility on the ground The on-board truss unit has been tested in both vibration and thermal vacuum conditions simulating orbit environment, and the ground teleoperation facility has been developed. The actual experiments will start in late 1997.
\end{abstract}

\section{Introduction}

Space robot systems are expected to play an important role for future large-scale structure construction on orbit. Developing a space station and a solar power satellite, telerobotic technology might become more essential on orbital activities than human astronauts for safety and economical reasons.

The primary goals, established in our research, are to develop telerobotic system concepts and to make research on technology related to the future orbital structures, such as robot-friendly space truss joint, space vision system and teleoperation with time-delay. Combining advanced space telerobotic technology with robot system research and construction experience on the ground, our unique approach for practical space robotic systems development has been started from 1991.

We are planning to make experiments in onorbit truss structure construction by using a manipulator teleoperated from the ground. The manipulator is mounted on Japanese Engineering Test Satellite No. 7 ( ETS-VII ) scheduled to be launched in 1997 by National Space Development Agency of Japan (NASDA). The truss experiment unit for robotic assembly, mounted also on ETSVII, facilitates two types of truss systems; an assembly truss joint and a deployable truss system.

In this paper we focus on the space construction concept, the telerobot construction experiment, and the mechanical design and test of the truss experiment unit.

\section{On-orbit construction concept}

\subsection{Design aspects for space environment}


On-orbit environment is much severer for space robots and astronauts comparing to the terrestrial construction outdoor environment for both human labors and robots. The space environment such as micro-gravity, vacuum and extreme thermal change has vital impacts for the space system design and development.

The absence of gravity makes system structures in space very different from those in the earth. Structures on the ground have the base for standing on the ground, however space structures have no base and floating, and have to be stabilized to keep its posture all the time. On the construction stage, terrestrial structures are generally constructed from the bottom to the top, while space construction system has to be from center to outward.

For the ground construction, gravity is the major external force to components handled by robots, while space robots keep inertial force of objects. Moreover there are no foundation in outer space to anchor the system. In order to assemble relatively large-size space structures, position and orientation of the structures must be cooperatively aligned to each other.

As rockets are the only available vehicles for space transportation, it is inevitable to apply extra severe vibration to payloads; the space structure components. As vibration load is much larger than that of transportation and construction on the ground, and it influences the design of the mechanical components of space structures. To survive under launching environments, special mechanisms are prepared for payloads, so called launch lock mechanism, locking the system during launch and releasing the system on orbit to work.

The mechanical design needs to take vacuum environment into account. The mechanical components such as hinges and joints utilize the space qualified lubrication in the vacuum condition. Gas outcoming in space must be avoided for contaminating the neighborhood components. Furthermore, extremely thermal change, radiation effects must be considered to design mechanical systems.

\subsection{On-orbit Construction Concept by Telerobot}

Limited launching capacity on dimensions and weight of the current vehicles makes the space assemble technology be vital for large scale space structures. Telerobotics will be one of key technologies to realize future space construction. We focus on truss joint assembly and truss deployment as the primary tasks for space robots because those tasks are considered to be two basic tasks in space structure construction.

In order for a space robot to manipulate a truss joint, consideration must be given to not only robot design but also joint design. We have developed a concept of the robot-friendly joint of truss structures for one hand robot arm. The joint must be operated with the minimum mechanical actions for simple manipulation. The force magnification mechanism inside the joint is required because of small handling force with large jointed structural strength. The centered mechanism and autoalignment of position while mating joint are also essential for inferior position accuracy of space robot.

Based on the system where space robots on orbit and control station on the ground, we consider the following three items as a technological breakthrough toward the large space system construction.

(1) Three dimensional space mobility either to walk on the truss structures or to fly around the structures

(2) Stable control under contact of objects and constraint by trajectories of deployment

(3) Teleoperation and autonomous control with image processing under communication time delay and narrow communication bandwidth

\section{Telerobotic Construction Experiment}

\subsection{Experiment Objective}

We have an opportunity to carry out space truss construction experiments by both the robot arm and the truss experiment unit mounted on ETS-VII. ETS-VII, scheduled to be launched in autumn of this year by NASDA, is the first satellite dedicated to space robot mission and rendezvous-docking mission in Japan. 
The ETS-VII orbit will be 550 kilometers in altitude and 35 degrees in inclination. The satellite composes two parts; Chaser satellite and Target satellite. And the robot arm and its experimental mission units are fixed on the chaser exterior panel facing to the earth.

In the space robot experiments, NASDA conducts the visual inspection and handling of the orbital replacement unit as on-orbit satellite servicing, and three Japanese national laboratories also participate their own experiments; advanced robot hand experiments (MITI-ETL); antenna assembling mechanism experiments (CRL); and truss structure handling experiments (NAL).

The space robot experiments will be mainly operated from the ground operation facility via data relay satellites such as Communication and Broadcasting Engineering Test Satellite ( COMETS ) located on geostationary orbit.

The objectives of our truss experiments are to establish basic technologies for on-orbit assembly and construction by telerobot. To perform truss assembly and deployment tasks by operating the robot arm from the ground, we have been developing the truss experimental unit mounted on ETS-VII and ground control station for handling the truss unit under teleoperation. In our experiments, we'll especially concentrate on the following four essential technologies.

(1)Manipulation of small objects by the space robot arm, utilizing the image processing.

(2)Teleoperation with constraint trajectory, utilizing the ground simulation.

(3)Demonstration of assembling and deploying tasks of the truss structure, using their major elements, such as latches, hinges, and joints.

(4)Smooth teleoperation under long communication time delay, using additional time delay method developed at NAL.

These technologies will be utilized in large space structure construction in the future.

\subsection{Truss Experiment System}

The experiment system consists of the truss structure handling unit and the ground teleoperation facility, besides NASDA's robot experiment facility as shown in Figure 1. Our ground teleoperation facility will be implemented just behind the NASDA's ETS-VII robot experiment operation facility. All telemetry data collected by ETS-VII, including the robot position and the truss unit status, will be downlinked to the teleoperation facility through the NASDA's facility. All of the teleoperation commands, generated from the operator console and joysticks on our facility, will be uplinked through the NASDA's facility to the ETS-VII robot arm, unless the teleoperation command will interfere with the interface and/or safety condition.

The twenty eight days are assigned for our experiments during one and half year ETS-VII mission life. The experiment days will be distributed among the whole mission life, and each experiment set composing two or three continuous days will mostly be available every month. One day consists of two or three passes, and each experiment pass continuing twenty minutes as real-time operation comes every ninety minutes. Such pass operation period is constraint due to ETS-VII orbit beta angle, COMETS communication linkage availability and ETS-VII optical condition for the robot cameras by albedo.

The experiment plan enables us to provide feedback to the rest of the experiment set in long term and to the next day in short term. Preparation of the own backup experiment pass, which is independent of the planed pass, avoids waste of valuable pass.

The experiment sets are divided into four categories depending on the level of the goals; initial checkout; minimum success; nominal success; and sufficient success. The relation of the level to our experiment contents are shown in Table 1. The experiment will shift gradually from the minimum to the sufficient success day by day.

\section{Truss Experiment Unit}

\subsection{ETS-VII Robot Arm Characteristics}

The ETS-VII robot arm is approximately a two meter long single manipulator with six degreesof-freedom, having a hand tool at the tip to grasp a 
grapple fixture. A CCD hand camera is mounted at the tip to provide a close view around the tool and to measure the relative tip position with respect to the grapple fixture. The monitor camera is also attached to the base of ETS-VII arm to show a global view of the truss unit.

The most inferior characteristic of the current space robots is their low level controllability. In the serious on-orbit environment, the control performance of the arm tip position is only ten millimeters in absolute accuracy, or two millimeters in relative accuracy, that are fifty to hundred times worse than usual industrial robots on the ground.

As a maximum generated operation force is only twenty newtons, the truss handling force must be designed within the range of the operation force. It also requires for truss system to sustain one hundred eighty newtons force for possible arm operation erros.

The time delay, due to communication between our ground teleoperation facility and robot on the satellite, makes teleoperation more difficult. Due to low communication bandwidth, the camera image is insufficient to process the image precisely.

\subsection{Truss Unit Design Principle}

The truss experiment unit is mounted on the ETS-VII satellite. The experiment unit, box-shaped with $380 \mathrm{~mm}$ width, $505 \mathrm{~mm}$ depth and $280 \mathrm{~mm}$ height, is shown in Figure 2. It consists of a truss joint assembly, a deployable truss, and a launch lock system for their truss components as shown in Figure 3. Each component has its own grapple fixture to be grasped by the robot hand tool, and its own target markers to measure relative position with respect to the robot hand by the camera on the arm tip.

The electrical parts including potentiometers and micro switches have been implemented to monitor the status of the truss unit on the ground. The heaters and thermostat have been also mounted on the truss unit to maintain its temperature.

Our truss unit design has been highly dependent on the characteristics of the robot arm. The design principles of the truss unit are to increase the mission success possibility of assembly and deployment tasks under such inaccurate arm control, and to increase the tolerance to prevent the truss unit from the mechanical damage in case of teleoperation error.

\subsection{Truss Joint Assembly Design}

The truss joint assembly will be jointed to the node by the robot arm. It is designed for the one hand robot arm to be assembled without any hand-over tasks to minimize mechanical actions.

The joint can be connected and released back and forth so that it can be operated under various experimental conditions. Because the jointing process is considered to be the most important in the whole truss assembly tasks, our truss joint is constraint on two dimensional plane and limited only to the mating process.

The jointed force is magnified by crank mechanism inside the joint which makes small handling force with large jointed structural strength possible. The mechanical guides in the joint allow the inaccurate arm to slide the joint, which is the most difficult in the truss assembly task.

In the case that the arm accidentally releases the joint during mating process, the joint will automatically return to the stowed position by the spring mechanism inside the joint without using the $\mathrm{arm}$. This function is necessary because the robot arm is only possible to grasp the fixture at the pre-determined location such as the stowed position with the target markers.

\subsection{Deployable Truss Structure Design}

The deployable truss structure composing truss struts connected by hinges will be handled to become a tetrahedral structure by the robot arm. The tetrahedral structure is selected as it has the minimum degrees-of-freedom among statically determinate truss structures.

The folding and latching function to deploy the structure is needed for only one strut over the three. During the deployment process no arm action is required to operate the latching mechanism, while the arm tip rotation initially needs to release the latch. 


\subsection{Launch Lock Mechanism Design}

The launch lock mechanism is required to fix the truss joint assembly mechanism and deployable truss structure during launch. It will release these trusses by the robot arm in the earlier phase of the on-orbit experiment.

One of challenges in designing our launch lock system is to release the two trusses simultaneously. Another difficulty is to release them within the available arm force and torque from the strong fixed force. The three stage sequential mechanism is employed to satisfy them as shown in Figure 4. The first stage is to magnify the torque and exchange to the force, the second to transfer the force and the third to distribute the force to the two. The mechanism makes it possible that the very low torque only of about one newton meter can easily release very strong force of about two handers newtons.

One of the concerns to employ the sequential mechanism is reliability. An unpredictable accident in either of the three stages during the sequences may disable not only launch lock system but also the whole function such as the truss joint assembly. The peg receptor is implemented in the second stage of the launch lock system so that the robot arm with the special tool peg, provided by ETS-VII system, can operate the slide action in the case of the first stage malfunction.

\subsection{Verification Test of Flight Model}

Any components of space system have to survive the severe environmental condition tests before launch. The vibration and shock impacts during launch, and extreme thermal change in vacuum condition on orbit are most serious for the mechanical components like our truss unit.

We have developed the flight model of the truss experiment unit, performing vibration test, thermal vacuum test and performance test. During the vibration test consisting of sinusoidal vibration and random vibration, twenty gravitational acceleration corresponding to the launching load is applied to the satellite interface base of the truss unit. Figure 5 shows the photograph of the vibration test view.

During the thermal vacuum test, the launch lock mechanism has been tested after being exposed to the less than minus thirty degree centigrade for more than twenty four hours, simulating on-orbit environment.

After these environment tests, the performance test including the measurement of the truss operating force has been performed. We have verified that the truss unit performance achieved the design requirement and no damage was found.

\section{Ground Teleoperation Facility}

We have designed the ground teleoperation facility to manipulate the truss unit by the robot arm. The teleoperation facility will have the following functions needed for the truss assembly experiments.

(1)Arm tip measurement by the ground image processing of the on-board target markers, using the arm tip camera.

(2)Pre-programmed hierarchical teleoperation with feedback control of the arm tip force sensor data and the image processing measurement, under the long communication time delay.

(3)Human interface for the joystick teleoperation to inform the operator with the telemetry data by the graphical user interface, to satisfy the safety operation requirements of the satellite.

(4)Computer graphics simulation of motion of the on-board robot arm and the truss unit, to verify the teleoperation commands in real-time against hazardous collision.

(5)Predicted display image and additional time delay method to compensate the communication time delay, and to increase teleoperation efficiency.

The teleoperation facility will consist of four workstations, three personal computers, and one industrial robot arm. The workstations will include two high speed graphical simulation computers, one image processing computer, and one man-machine-interface computer. Almost all information will be gathered to the man-machineinterface workstation for a safety-operation. All the computers are connected as a eather-net local area 
network.

This facility will be used not only for the actual teleoperation, but also for preparation of the experiment itself, such as the design of sequence of event and the validation of command sequence, before the real teleoperation experiments of the robot arm.

\section{Conclusion}

On-orbit assembly is one of the key technologies for future space construction. We will conduct the space truss construction experiments by ETS-VII robot arm remotely operated from the ground. The on-board truss unit on ETS-VII has been verified and the teleoperation facility has been developed.

After the ETS-VII launch in autumn 1997, the experiment mission will continue for one and half years. Through this mission period, we hope to achieve all of our experiment purposes, in cooperating with other three agencies.

\section{Reference}

[1]Wendel, T. Yoshida, K. Takagi, "Truss Joint System for Space Structures", Proceedings of 16th International Symposium on Space Technology and Science, Sapporo, 1988
[2]Construction Robot Study Group, "Construction Robot", Kajima Pub., 1990, (In Japanese)

[3]H. Ueno, T. Yoshida, S. Kibe and K. Matsumoto, "Study on Joint Mechanism for Truss Structure Assembly by Space Robot", Proceedings of 37th Space Sciences and Technology Conference, Kitakyushu, 1993 (in Japanese)

[4]K. Matsumoto, S. Kibe, I. Yamaguchi, T. Kida, H. Ueno, H. Satoh, S. Aoki and T. Yoshida, " Truss Structure Tele-Manipulation Experiment using ETS-7" , Proceedings of 3rd i-SAIRAS-94, Pasadena, 1994

[5]K. Matsumoto, S. Kibe, S. Wakabayashi, H. Ueno, H. Satoh, S. Aoki and T. Yoshida, "Development of BBM of Truss Structure Experiment Unit on ETS-7, Proceedings of 39th Space Sciences and Technology Conference, Osaka, 1995 (in Japanese)

[6] K. Matsumoto, S. Wakabayashi, H. Satoh, H. Ueno, S. Aoki and T. Yoshida, “ Development of Proto Flight Model for ETS-VII Truss Structure", Proceedings of 40th Space Sciences and Technology Conference, Morioka, 1996 (in Japanese)

Table 1 Truss Experiment Category

\begin{tabular}{|c|c|c|c|c|c|}
\hline Exionition & $\begin{array}{l}\text { Maniputation of small } \\
\text { Objects }\end{array}$ & trajecton: & constrantsing & $\begin{array}{l}\text { Demonstration of assembling and } \\
\text { deploying tasks }\end{array}$ & 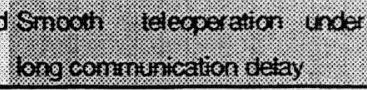 \\
\hline antisis: & $\begin{array}{l}\text { - launch lock release } \\
\text { - markers image processing } \\
\text { - robot arm operation }\end{array}$ & & & & 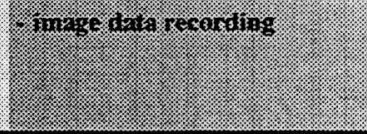 \\
\hline$(\sin 20$ & $\begin{array}{l}\text { - grapple fixtures grasping } \\
\text { - markers image processing } \\
\text { - backlash modeling }\end{array}$ & 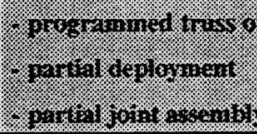 & $\cos =1501$ & $\begin{array}{l}\text { - truss joint assembly } \\
\text { - truss structure deployment }\end{array}$ & \\
\hline 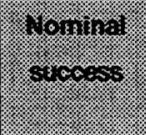 & & 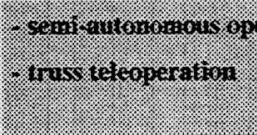 & tration & $\begin{array}{l}\text { - truss joint stowed } \\
\text { - truss undeployment }\end{array}$ & 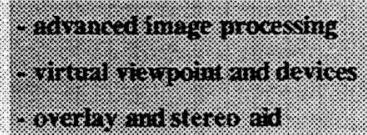 \\
\hline 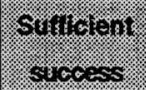 & - hand tool back-driving & 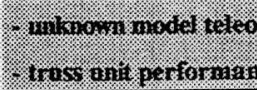 & $\cos$ incasing & $\begin{array}{l}\text { - quick joint assembly } \\
\text { - quick truss deployment }\end{array}$ & 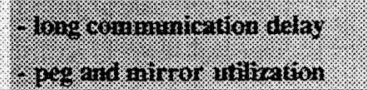 \\
\hline
\end{tabular}




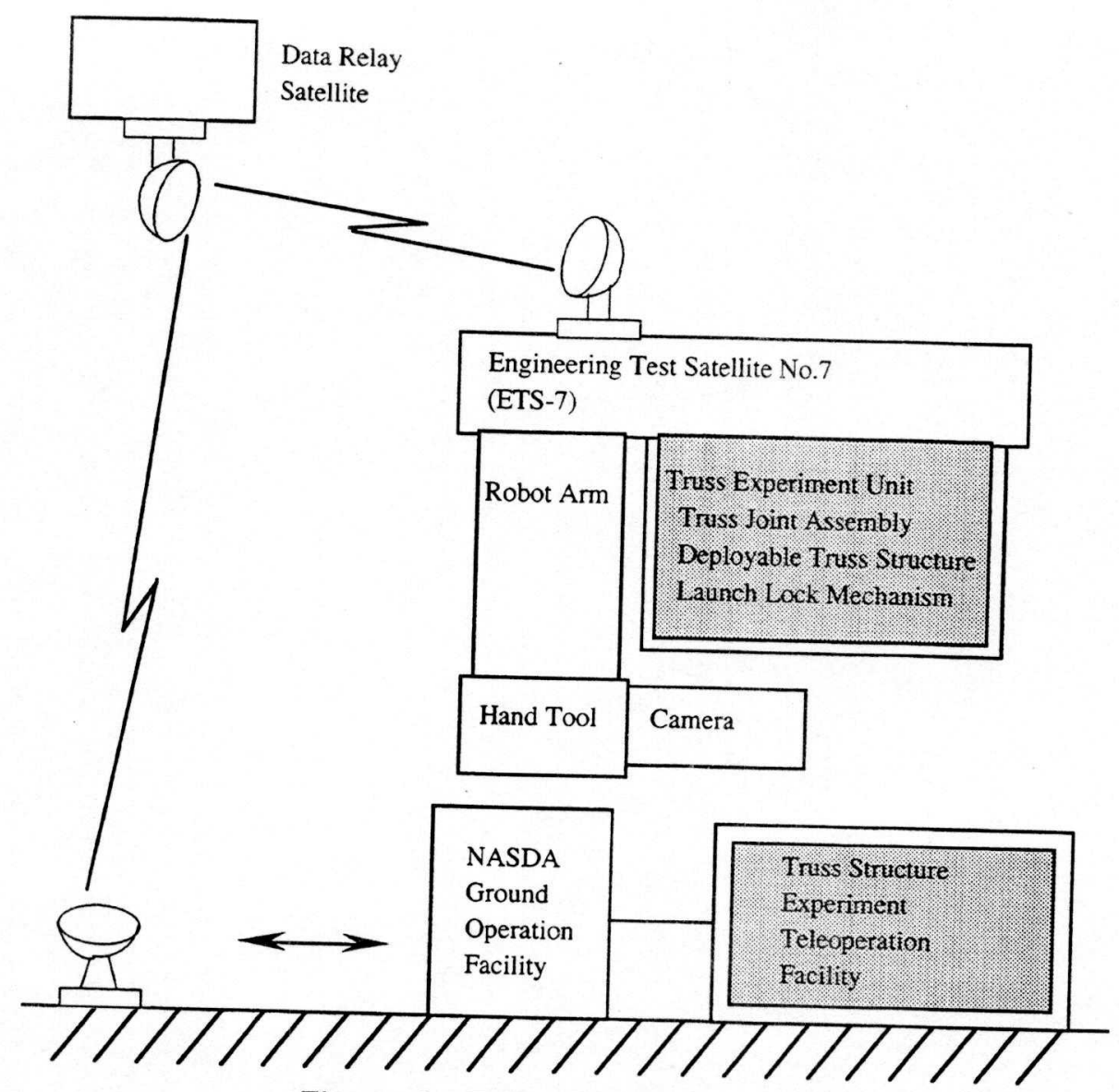

Figure 1 Truss Experiment System

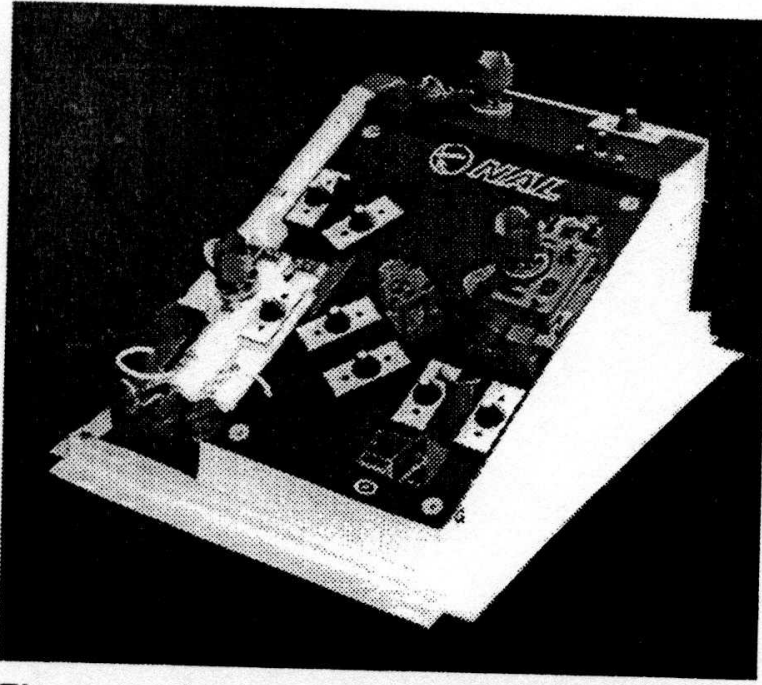

Figure 2 (1) Photo of Truss Experiment Unit (Stowed Configuration)

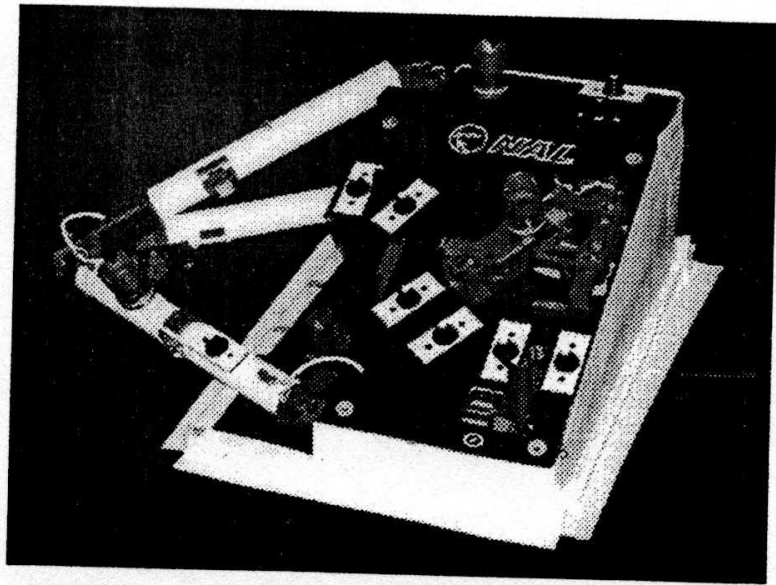

Figure 2 (2) Photo of Truss Experiment Unit (Assembled and Deployed Configuration) 


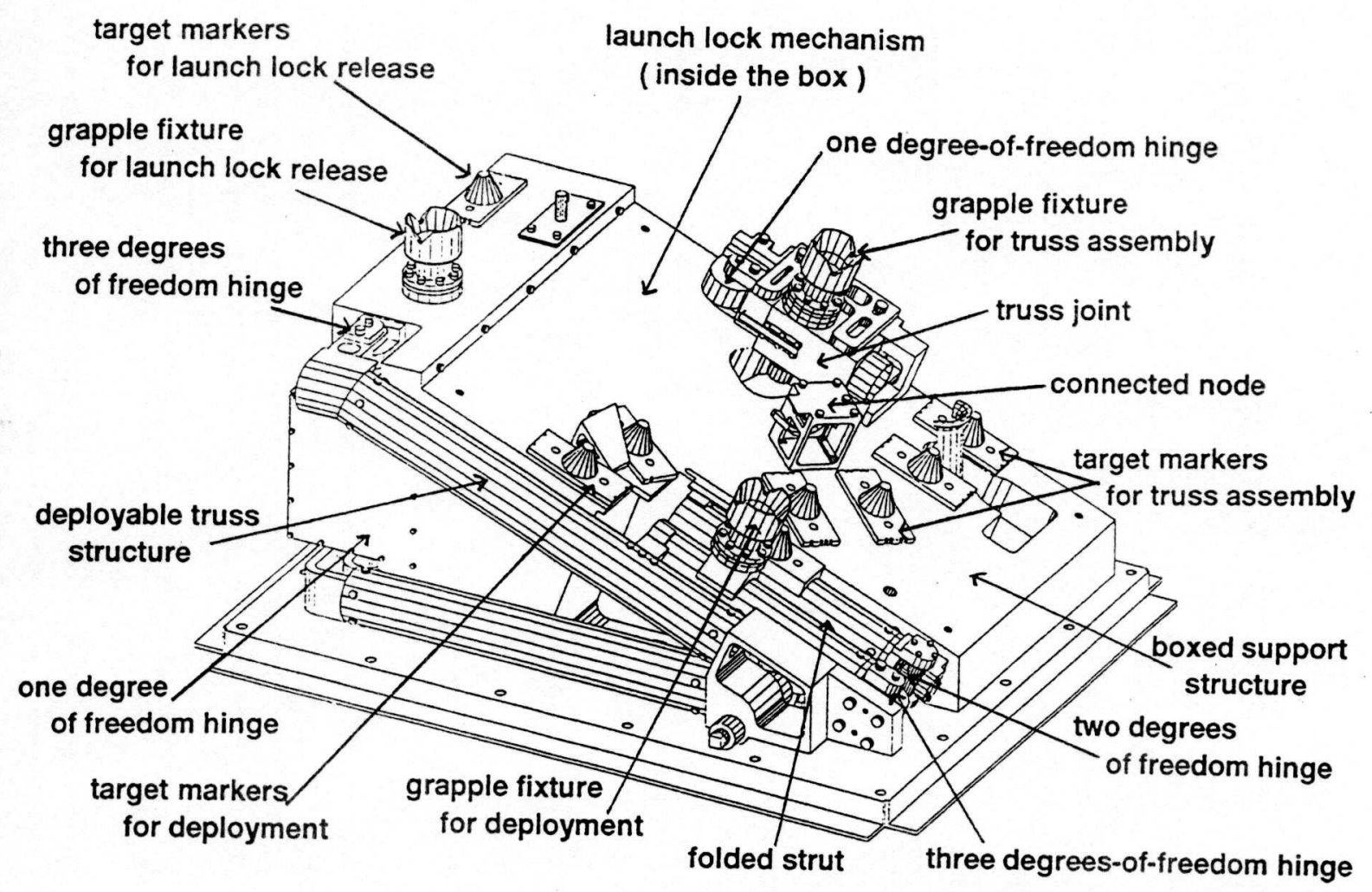

Figure 3 Truss Experiment Unit Component

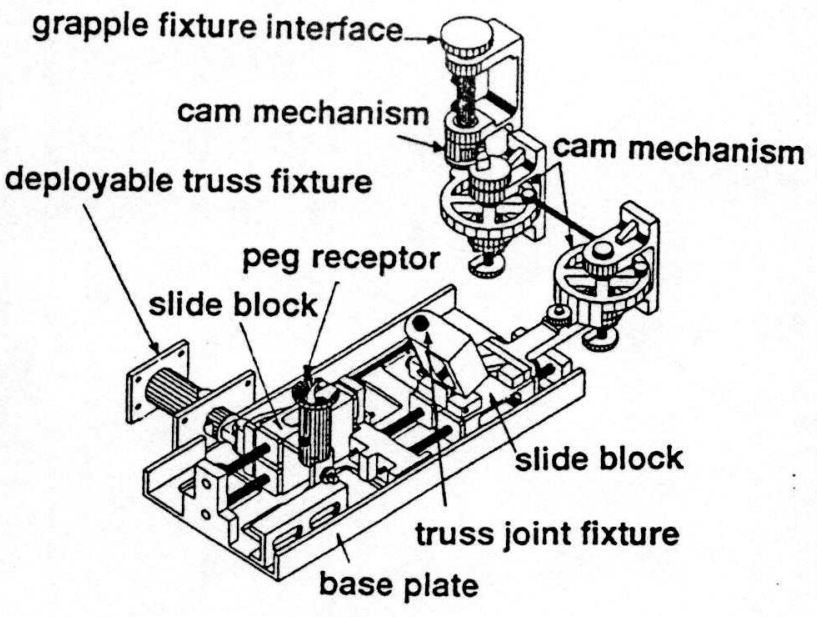

Figure 4 Launch Lock Mechanism

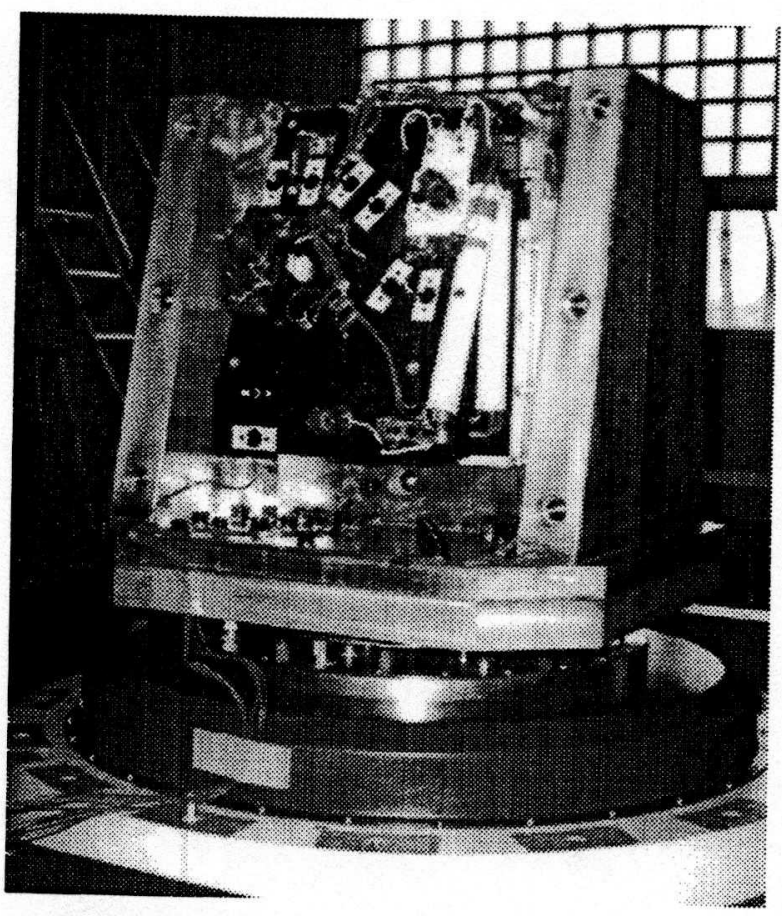

Figure 5 Photo of Vibration Test View 